\title{
GROUPS WITH AN IRREDUCIBLE CHARACTER OF LARGE DEGREE ARE SOLVABLE
}

\author{
FRANK DEMEYER
}

\begin{abstract}
The degree of an irreducible complex character afforded by a finite group is bounded above by the index of an abelian normal subgroup and by the square root of the index of the center. Whenever a finite group affords an irreducible character whose degree achieves these two upper bounds the group must be solvable.
\end{abstract}

Let $G$ be a finite group with an irreducible (complex) character $\zeta$. If $Z$ is the center of $G$ it is easy to prove that $\zeta(1)^{2} \leqq[G: Z]$ and if $A$ is an abelian normal subgroup of $G$ it is easy to show that $\zeta(1) \leqq[G: A]$ (see pp. 364-365 of [1]). Say the group $G$ admits an irreducible character of large degree if $\zeta(1)^{2}=[G: A]^{2}=[G: Z]$, that is whenever the two bounds noted above are achieved simultaneously. Such groups arise in the theory of projective representations and the Galois theory in general rings [2]. The purpose of this note is to give proof of the result stated in the title, thus verifying a special case of a conjecture in [2]. Throughout all unexplained terminology is as in [1].

Theorem. Let $G$ be a group with center $Z$ and abelian normal subgroup $A$ so that there is an irreducible complex character $\zeta$ on $G$ with $\zeta(1)^{2}=[G: A]^{2}=[G: Z]$. Then $G$ is solvable.

Proof. A theorem of P. Hall (Theorem 4.5, p. 233 of [3]) asserts that a group is solvable if and only if every $p$-sylow subgroup has a complement. This theorem will be applied to $G / A$ to give the result.

Since the degree of any irreducible character is bounded by the index of an abelian subgroup, $A$ is a maximal abelian normal subgroup of $G$, so $Z \subseteq A$. If $\pi, \Pi$, and $P$ are sylow $p$-subgroups of $Z, A$, and $G$ respectively then $\pi \subseteq \Pi \subseteq P$. Moreover $\pi$ is contained in the center of $P, \Pi$ is an abelian normal subgroup of $P$, and $\Pi$ is a normal subgroup of $G$.

Arguing as in [2] we show $\left.\zeta\right|_{P}=m \lambda$ where $\lambda$ is irreducible on $P$ and $\lambda(1)=[P: \Pi]$. By Schur's lemma we have $\left.\zeta\right|_{z}=\zeta(1) \phi$ for some linear character $\phi$ of $Z$. Then $\left(\zeta, \phi^{G}\right)=\left(\left.\zeta\right|_{z}, \phi\right)=\zeta(1)$ so by counting degrees $\zeta(1) \zeta=\phi^{G}$. Let $R$ be the subgroup of $G$ generated by $Z$ and $P$, and let $\lambda$ be an irreducible character of $R$ contained in $\phi^{R}$. By Schur's

Received by the editors December 29, 1969.

AMS Subject Classifications. Primary 2080; Secondary 2027, 1670.

Key Words and Phrases. Finite group, irreducible complex character, solvable group. 
lemma $\left.\lambda\right|_{P}$ remains irreducible because the elements of $Z$ are represented by scalars. Since $\lambda$ is contained in $\phi^{R}, \lambda^{G}=m \zeta$ for some integer $m$. By counting degrees

$$
m=[G: R] \lambda(1) / \zeta(1) .
$$

Since $\lambda$ is irreducible on $P, \lambda(1)=p^{a}$ for some $a ;[G: R]$ is prime to $p$ since $R$ contains $P$; the $p$-part of $\zeta(1)^{2}$ is $[P: P \cap Z]$ and $P \cap Z=\pi$. Thus we have shown $\lambda(1)^{2}=[P: \pi]=(P: \Pi)^{2}$ and $\left.\zeta\right|_{P}=m \lambda$. Now by Cliffords theory (p. 343 of $[1]$ )

$$
\left.\lambda\right|_{\mathrm{II}}=\alpha^{1}+\alpha^{2}+\cdots+\alpha^{n}
$$

where the $\alpha^{i}$ are conjugate linear characters on $\Pi$ (conjugate by elements in $P$ ). Let $\alpha=\alpha^{1}$ and $g \in G$, it follows that $\alpha^{(g)}$ is contained in $\left.\lambda^{(g)}\right|_{\mathrm{I}}$ which in turn is contained in $\left.\zeta^{(\theta)}\right|_{\mathrm{I}}=\left.\zeta\right|_{\Pi}=\left.m \lambda\right|_{\Pi}$. Thus $\alpha^{(g)}=\alpha^{i}$ for some $i$, and every $G$-conjugate of $\alpha$ is a $P$ conjugate of $\alpha$. To determine $n$ observe that the $p$-part of $\zeta(1)$ is $[P: \Pi]=\lambda(1)=n$ which is the $p$-part of $[G: A]$. Let $H^{*}=\left\{g \in G \mid \alpha^{(g)}=\alpha\right\}$, then $H^{*}$ is the inertia group of $\alpha$ in $G$ and $\left[G: H^{*}\right.$ ] is the number of conjugates of $\alpha$ (p. 346 of $[1])$. Thus $\left[G: H^{*}\right]$ is the $p$-part of $[G: A]$ so $H^{*} / A$ is a $p$-complement of $P / A$ in $G / A$ which proves the theorem.

To show that the hypothesis on the center $Z$ of $G$ is necessary for the theorem let $H$ be any group and let $J_{p}(H)$ be the group algebra of $H$ over the field with $p$-elements ( $p$ any prime). Let $A=J_{p}(H)$ viewed as an additive group and let $H$ act as a group of automorphisms of $A$ by

$$
h(a x)=a h x \quad \text { (regular representation) } x, h \in H, a \in J_{p} .
$$

Let $G$ be the semidirect product of $A$ by $H$ with respect to this representation of $H$ as automorphisms of $A$. Then $A$ is an abelian normal subgroup of $G$. Let $\theta$ be the linear character on $A$ defined by $\theta(a x)$ $=\xi^{a} \delta_{x, 1}$ where 1 is the identity in $H, \xi$ is a primitive $p$ th root of 1 , and $a$ is the least positive integer representing the corresponding class in $J_{p}$. It is easy to see that $\theta$ has $[H: 1]$ distinct conjugates under the action of $G$ so $\theta^{G}$ is irreducible and $\theta(1)=[G: A]$. Observe that $H$ $=G / A$ is arbitrary in this construction.

It is also easy to observe that if $G$ is a group satisfying all the hypotheses of the theorem and if we let $H=G / A$ then the natural semidirect product of $A$ by $H$ also satisfies the hypotheses of the theorem.

There is a central extension of $A_{4} \times C_{3}\left(A_{4}\right.$ the alternating group of order $12, C_{3}$ the cyclic group of order 3 ) which is a group with an irreducible character of large degree, this group is not nilpotent. 
Using [2] (Theorem 1) the relationship between the groups we are studying, projective representations, and the Schur multiplier can be pointed out. If $\alpha$ is in the Schur multiplier of the group $G$ and $K$ is the complex field then $(K G)_{\alpha}$ denotes the corresponding projective group algebra.

CoROLlary. There is a group $H$ and an element $\alpha$ in the Schur multiplier of $H$ so that $(K H)_{\alpha}$ has center $K$ and an abelian normal subgroup $A$ of $H$ with $[H: A]^{2}=[H: 1]$ if and only if there is a central extension $G$ of $H$ satisfying the hypothesis of the theorem.

Proof. The corollary is immediate on combining the theorem and Theorem 1 of [2].

\section{BIBLIOGRAPHY}

1. C. W. Curtis and I. Reiner, Representation theory of finite groups and associative algebras, Pure and Appl. Math., vol. XI, Interscience, New York, 1962. MR 26 \#2519.

2. F. R. DeMeyer and G. J. Janusz, Finite groups with an irreducible representation of large degree, Math. Z. 108 (1969), 145-153. MR 38 \#5910.

3. D. Gorenstein, Finite groups, Harper, New York, 1968. MR 38 \#229.

Colorado State University, Fort Collins, Colorado 80521 